\title{
Response to comment to "Magnetic resonance imaging findings of bilateral thalamic involvement in severe paroxysmal sympathetic hyperactivity: a pediatric case series"
}

\author{
Serge Mrkobrada ${ }^{1}$
}

Received: 29 December 2015 / Accepted: 4 January 2016/Published online: 11 January 2016

(C) Springer-Verlag Berlin Heidelberg 2016

\section{Dear Editor:}

Dr. Shukla's comment points out limitations of our study [1], including the small sample size of three patients. We recognize this limitation within our manuscript and agree that larger cohorts are needed to prove our hypothesis of bilateral thalamic involvement in severe paroxysmal hyperactivity (PSH) in patients with hypoxemic brain injury.

We also agree that the pathophysiology of PSH is very complex. We do not argue that the only neuroanatomical basis for developing severe PSH is bilateral thalamic involvement, and there may be others, as pointed out by Dr. Shukla. However, in severe hypoxemic brain injury, our case series suggests that bilateral thalamic involvement may be an important marker of severe PSH. To support our hypothesis, we outlined both studies on animal models as well as fatal familial insomnia that have implicated the role of the thalamus in autonomic control [2-4]. Interestingly, the clinical manifestations of fatal familial insomnia are similar to PSH, a disease that is characterized by severe degeneration of bilateral mediodorsal and anterior thalamic nuclei with sparing of other autonomic centers $[2,3]$. In publishing this hypothesis, it was our hope to stimulate further research and discussion of PSH, which we are pleased to see, has already happened.

\section{Compliance with ethical standards}

Conflict of interest The author declares that there are no conflicts of interest.

\section{References}

1. Shukla D (2015) Comment to "Magnetic resonance imaging (MRI) findings of bilateral thalamic involvement in severe paroxysmal sympathetic hyperactivity (PSH): a pediatric case series". Child's Nerv Syst. published online December 11, 2015

2. Benarroch EE, Stotz-Potter EH (1998) Dysautonomia in fatal familial insomnia as an indicator of the potential role of the thalamus in autonomic control. Brain Pathol 8:527-530

3. Lugaresi E, Medori R, Montagna P et al (1989) Fatal familial insomnia and dysautonomia with selective degeneration of thalamic nuclei. N Engl J Med 315(16):997-1003

4. Stotz-Potter E, Benarroch E (1998) Removal of GABAergic inhibition in the mediodorsal nucleus of the rat thalamus leads to increases in heart rate and blood pressure. Neurosci Lett 247:127-130
Serge Mrkobrada

mrkobrada@gmail.com

Calgary, AB, Canada 